\title{
RELATION BETWEEN CHLAMYDIA TRACHOMATIS, THE TROPHOBLAST AND PRETERM DELIVERY
}

\author{
Emil Kovachev, Stephan Ivanov, Blagovest Bechev, Nikolay Kolev, Stephan Kisyov, \\ Simona Anzhel
}

Department of Obstetrics and Gynecology, Medical University of Varna

\begin{abstract}
There is a connection between preterm labor and intrauterine infections which is wellestablished in up to $40 \%$ of cases. Success of the interaction between the invading trophoblast at the maternal-fetal interface and the maternal immune system is an immunologically unique fact. In this process the trophoblast participates in both the immune suppression, which is necessary for tolerance to the semialogenic fetus, and the immune activation against damaging factors such as infections. Alterations in this type of cross-talk, as in the cases of infection-triggered inflammation by Chlamydia species, could result in pregnancy complications such as prematurity or spontaneous abortion.
\end{abstract}

Related articles and clinical cases have been gathered and reviewed to establish the main mechanisms and to confirm the relationship between preterm labor and Chlamydia trachomatis infection.

Keywords: genital chlamydiosis, toll-like receptors, heat shock protein, pregnancy

\section{INTRODUCTION}

There are many debates about the role of the immune system in realizing or obstructing a normal pregnancy and how the human endometrium becomes an immunologically unique place - on the one hand it simultaneously tolerates the semiallogenic fetus and placenta, but, on the other hand, it maintains the protection against foreign antigens. It is thought that the trophoblast at the maternal-fetal interface is the structure that actively participates in preventing allorecognition and in the response to pathogens that may compromise the fetal well-being. The relation between preterm labor and intrauterine infections is well established up to $40 \%$ of cases. (12)

Address for correspondence:

Emil Kovachev MD,

SBAGAL "Prof. d-r D. Stamatov"

150, Tzar Osvoboditel str.,Varna, Bulgaria

Phone: + 359898461288

e-mail: kovachev_md@abv.bg

Received: December 22, 2015

Accepted: February 15, 2016

\section{THE TROPHOBLAST}

The trophoblast, just like any innate immune cell, expresses pattern receptors that function as 'sensors' of the surrounding environment $(1,2)$. They act in recognition of bacterial and viral antigenes and damaged tissues. If the receptors are activated, the trophoblast will secrete a specific set of cytokines that, in turn, will act based upon the immune cells within the decidua (i.e. macrophages, $\mathrm{T}$ regulatory cells, NK cells), teaching them to work together in aid of the growing fetus. The major group is the toll-like receptor family (TLR). These are transmembrane proteins which individually respond to limited ligands but collectively act with different kinds of proteins. Studies in term placenta have demonstrated the exact functions of TLR-2 and TLR-4 $(3,7)$. TLR2 recognizes bacterial lipoproteins, peptidoglycan (PDG) and lipoteichoic acid (LTA) (16), while TLR-4 recognizes Gram-negative bacterial LPS (14). It is established that TLR-4 ligation promotes cytokine production, while TLR-2 directly activates apoptosis in first trimester trophoblast cells. These findings sug- 
Emil Kovachev, Stephan Ivanov, Blagovest Bechev et al.

gest that activation of TLR- 4 by microbal LPS leads to an increased production of both pro- and anti-inflammatory cytokines, especially IL-6 and IL-8, and, while this process does not directly induce trophoblast cell death, it provides an alternative mechanism for trophoblast cell damaging by inducing TNFa and IFNa expressions.

\section{CHLAMYDIA TRACHOMATIS INFECTION}

The most common reason for tubal infertility is the Chlamydia trachomatis $(\mathrm{Ct})$ infection. In addition, there is growing evidence to suggest that a Ct infection may also be associated with pregnancy complications, such as stillbirth, spontaneous abortion and prematurity $(4,15)$. C. trachomatis can infect a wide range of cell types, including the epithelial cells of the eye and the genital tract, the monocytes, and the fibroblasts. Furthermore, clinical studies have demonstrated that $\mathrm{Ct}$ can infect the placenta and decidua $(5,10)$.

C. trachomatis is an obligate, gram-negative intracellular bacteria which develops in eukaryotic cells. Altough it has its own metabolic system, Chlamydia is unable to produce adenosine-triphosphate (ATP), so it uses the amount present in the host cell. It has a unique biphasic life cycle, which is comprised of two types of reproduction - the extracellular phase of infectious elementary bodies (EB) during which it binds to and invades the epithelial cells through a receptor - mediated endocytosis, and the intracellular phase of metabolically active non-infectious replication competent reticular bodies (RB). Once inside the cytoplasm of the target cell, the EB converts into a reticular body. RB replication occurs within a specialized vacuole, known as an inclusion form (6). Following replication, the RBs convert into EBs, at which time the infected cell already contains up to 1000 infectious cells (30-40 hours) which then get released from the host cell, either by cell lysis or by extrusion of the inclusion, to infect neighboring cells (14). During an infection, Chlamydia modifies the host cell by secreting virulence factors into the cellıs cytoplasm using a type III secretion system. Some of these factors prevent fusion of the inclusion with the cell's lysosomes and block apoptosis, while other factors act as proteases - such as CPAF which degrades transcription factors important for the upregulation of MHC class I and class II and keratin.
Clinical studies have strongly suggested a link between bacterial infections with preterm labor (11). Inflammatory - induced excessive cytokine secretion at the maternal-fetal interface is thought to be a main contributor to a compromised pregnancy. One hypothesis as to how this reaction arises, is that through the expression of the toll-like receptors, the trophoblast cells have the ability to recognize and respond to microorganisms that may pose a threat to the embryo and pregnancy outcome. Since the interaction between the maternal immune system and the invading trophoblast at the fetal-maternal interface may be crucial for a successful pregnancy, alterations in this type of cross-talk, as in the case of infectiontriggered inflammation by Chlamydia species, could result in a complicated pregnancy (19).

Chlamydiae express a variety of ligands that could serve as potential TLR activators. LPS appears to utilize TLR4 for signaling, although it may be more dependent on the CD14 membrane than it has been reported for other species of LPS. Another important chlamydial antigen is the heat shock protein. When $\mathrm{Kol}$ and colleagues examined the proinflammatory activity of purified chlamydia HSP60 (cHSP60) preparations, they found that it resembled that of LPS, including the activation of the transcription factor NF$\kappa \mathrm{B}$, activation of the p38 mitogen-activated protein kinase, and the induction of proinflammatory cytokines (20). Several groups subsequently reported that cHSP60 could activate TLR2 and/or TLR4.

Normally, during the pre-and peri-implantatory phase of pregnancy, the embryo and decidua secrete proinflammatory and anti-inflammatory cytokines like TNFx, IL, growth factors, heat shock proteins, mainly Hsp60 and 70 and other products which proves that the success of implantation is secondary to the development of an injury-induced inflammatory reaction between the invading blastocyst and the endometrium (Fig. 1).

The implantation is an injury-induced inflammatory reaction by the natural killer cells, macrophages and T cells. Fetally derived trophoblast cells are in contact with maternal immune cells at two sites: villous trophoblasts are surrounded by maternal immune cells in the intervillous space and invading extravillous trophoblasts interact with decidual leukocytes. 

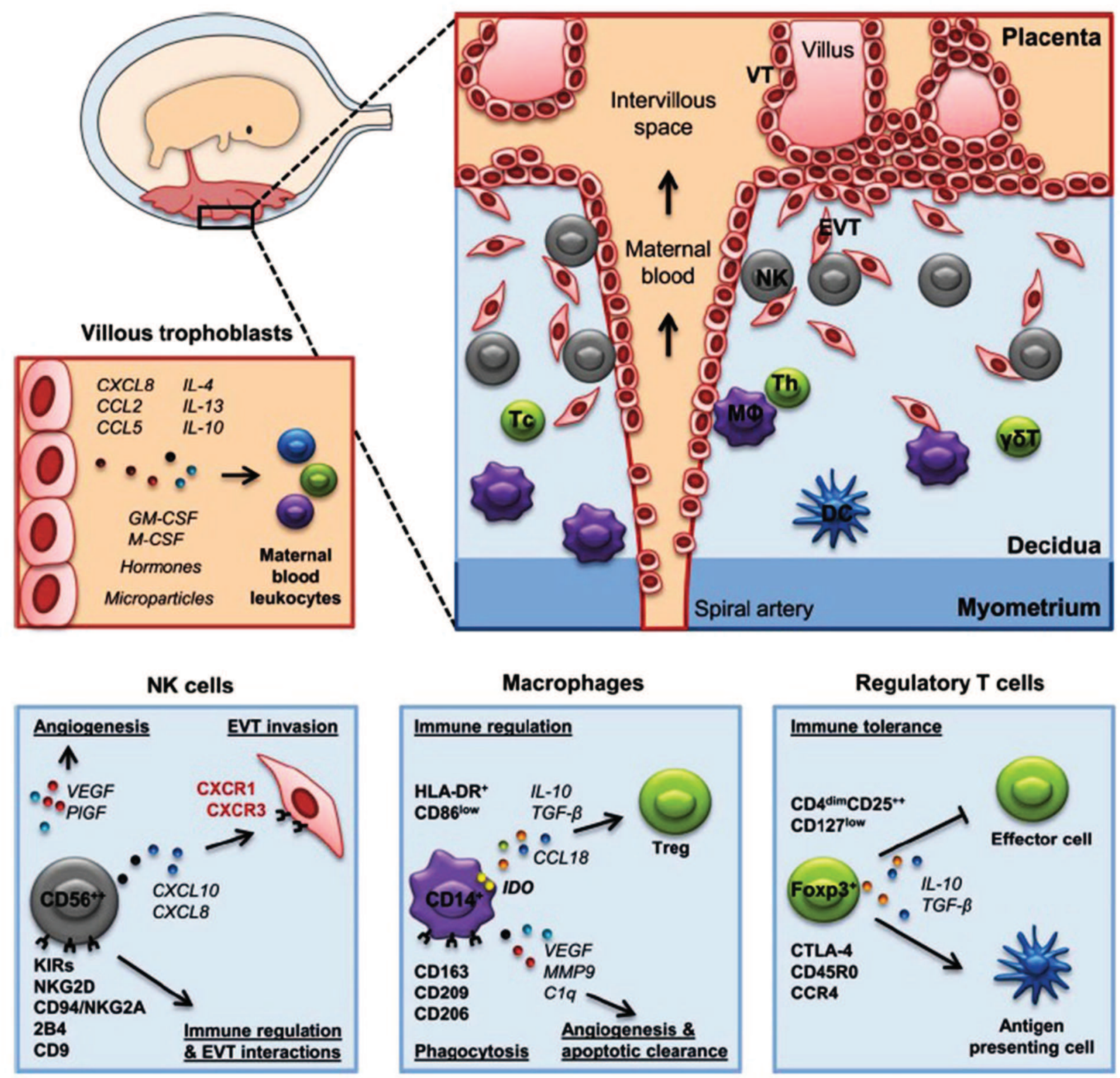

Fig. 1. The fetal-maternal interface.

The implantation is an injury-induced inflammatory reaction by the natural killer cells, macrophages and T cells. Fetally derived trophoblast cells are in contact with maternal immune cells at two sites: villous trophoblasts are surrounded by maternal immune cells in the intervillous space and invading extravillous trophoblasts interact with decidual leukocytes. (Source: Svensson - Arvelund et al. - Toxicologic Pathology, 42: 327-338, 2014).

A latent infection in the endometrium, as chronic chlamydiosis can totally deregulate and damage the process, by inducing hypersecretion of other cytokines such as ILl, IL8, IL6 and CSF strongly induce pathological T-cell and macrophage activation and cytokine production. Therefore, cytokines secreted by infection-activated immune cells send a wrong message to the developing embryo and de- stroy the balance between the pro and the anti-inflammatory activity, resulting in disrupting the embryo growth and suppressing its rejection by the maternal tissue.

Studies have consistently shown that there is a correlation between the production of chlamydial 60-kDa heat shock protein (Hsp60) antibodies in 
Emil Kovachev, Stephan Ivanov, Blagovest Bechev et al.

chlamydia-infected patients and adverse disease consequences. Hsp60 interacts with toll-like receptor 4, which stimulates the proliferation of human vascular smooth muscle cells and activates macrophages and endothelial cells (17). In both caspase-dependent and caspase-independent pathways, this interaction also leads to apoptosis of placental fibroblasts and trophobalst cells. Bacterial invasion of the choriodecidual space, acting in part through release of endotoxins and exotoxins, activates the decidua and the fetal membranes to produce a number of cytokines, including tumor necrosis factor $\alpha$, interleukin$1 \alpha$, interleukin- $1 \beta$, interleukin- 6 , interleukin-8, and the granulocyte colony-stimulating factor $(8,9,13,18)$. Furthermore, these cytokines stimulate prostaglandin synthesis and also initiate neutrophil chemotaxis, infiltration, and activation, culminating in the synthesis and release of metalloproteases and other bioactive substances. The prostaglandins stimulate uterine contractions while the metalloproteases attack the chorioamniotic membranes, leading to rupture. The metalloproteases also remodel the collagen in the cervix and soften it. As a result, these interactions lead to premature rupture of the amniotic membrane and preterm labor.

\section{CONCLUSION}

Chlamydia trachomatis is the most common bacterial sexually transmitted disease. As the majority of women are asymptomatic they are often unaware that they are infected. Therefore, Chlamydia can have a serious impact on a women's reproductive potential, with $40 \%$ of the cases leading to a pelvic inflammatory disease. About $5-8 \%$ of them become infertile or may have an ectopic pregnancy. Even if a woman gets pregnant, a latent Ct infection is a potential risk for pregnancy complications.

\section{ACKNOWLEDGMENTS}

We gratefully acknowledge the contribution of the Medical University of Varna. The authors of the article have declared that no conflict of interest exists.

\section{REFERENCES}

1. Abrahams VM, Romero R, Mor G. TLR-3 and TLR-4 mediate differential chemokine production and immune cell recruitment by first trimester tro- phoblast cells. Am J Reprod Immunol 2005; 53: 279. ASRI205-202.

2. Abrahams VM, Visintin I, Aldo PB, Guller $\mathrm{S}$, Romero R, Mor G. A role for TLRs in the regulation of immune cell migration by first trimester trophoblast cells. J Immunol 2005; 175: 8096-8104.

3. Abrahams VM, Mor G. Toll-like receptors and their role in the trophoblast. Placenta. 2005;26:540-547

4. Baud D, Regan L, Greub G. Emerging role of Chlamydia and Chlamydia-like organisms in adverse pregnancy outcomes. Curr Opin Infect Dis. 2008;21:70-76.

5. Baboonian C, Smith DA, Shapland D, Arno G, Zal B, Akiyu J, Kaski JC. Placental infection with Chlamydia pneumoniae and intrauterine growth restriction. Cardiovasc Res. 2003;60:165-169.

6. Beagley KW, Timms P. Chlamydia trachomatis infection: incidence, health costs and prospects for vaccine development. J Reprod Immunol. 2000;48:47-68.

7. Bulut, Y., E. Faure, L. Thomas, H. Karahashi, K. S. Michelson, O. Equils, S. G. Morrison, R. P. Morrison, and M. Arditi. 2002. Chlamydial heat shock protein 60 activates macrophages and endothelial cells through Toll-like receptor 4 and MD2 in a MyD88-dependent pathway. J. Immunol. 168:1435-1440.

8. Cassell G, Andrews W, Hauth J, et al. Isolation of microorganisms from the chorioamnion is twice that from amniotic fluid at cesarean delivery in women with intact membranes. Am J Obstet Gynecol 1993;168:424-424 abstract.

9. Dudley DJ. Pre-term labor: an intra-uterine inflammatory response syndrome? J Reprod Immunol 1997;36:93-109

10. Dong ZW, Li Y, Zhang LY, Liu RM. Detection of Chlamydia trachomatis intrauterine infection using polymerase chain reaction on chorionic villi. Int J Gynaecol Obstet.1998;61:29-32

11. Goncalves, L. F., T. Chaiworapongsa, R. Romero. 2002. Intrauterine infection and prematurity. Ment. Retard. Dev. Disabil. Res. Rev. 8:3.

12. Goldenberg, R. L., J. C. Hauth, W. W. Andrews. 2000. Intrauterine infection and preterm delivery. N. Engl. J. Med. 342:1500.

13. Gomez R, Romero R, Edwin SS, David C. Pathogenesis of preterm labor and preterm premature rupture of membranes associated with in- 
traamniotic infection. Infect Dis Clin North Am1997;11:135-176

14. Hossain A, Arif M, Ramia S, Bakir TF. Chlamydia trachomatis as a cause of abortion. J Hyg Epidemiol Microbiol Immunol. 1990;34:53-55.

15. Jain A, Nag VL, Goel MM, Chandrawati, Chaturvedi UC. Adverse foetal outcome in specific IgM positive Chlamydia trachomatis infection in pregnancy. Indian J Med Res. 1991;94:420-423

16. Lien, E., T. J. Sellati, A. Yoshimura, T. H. Flo, G. Rawadi, R. W. Finberg, J. D. Carroll, T. Espevik, R. R. Ingalls, J. D. Radolf, D. T. Golenbock. 1999. Toll-like receptor 2 functions as a pattern recognition receptor for diverse bacterial products. J. Biol. Chem. 274:33419.

17. Manavi K. A review on infection with Chlamydia trachomatis. Best Pract Res Clin Obstet Gynaecol.2006;20:941-951

18. Mitsunari M, Yoshida $S$, Shoji $T$ et al. Macrophage-activating lipopeptide-2 induces cyclooxygenase- 2 and prostaglandin $\mathrm{E}(2)$ via toll-like receptor 2 in human placental trophoblast cells. J Reprod Immunol 2006; 72: 46-48

19. Mor G, Romero R, Aldo PB, Abrahams VM. Is the trophoblast an immune regulator? The role of Toll-like receptors during pregnancy. Crit Rev Immunol. 2005;25:375-388.

20. Ohashi, K., Burkart, V., Flohe, S., and Kolb, H. (2000) J. Immunol. 164, 558-561 